\section{Urologia \\ Internationalis}

Urol Int 2011;87:42-48

DOI: $\underline{10.1159 / 000325463}$
Received: December 1, 2010

Accepted after revision: February 14, 2011

Published online: June 9, 2011

\title{
Concomitant Carcinoma in situ in Cystectomy Specimens Is Not Associated with Clinical Outcomes after Surgery
}

\author{
Philipp Nuhn ${ }^{a}$ Patrick J. Bastian ${ }^{a, b}$ Giacomo Novara ${ }^{c}$ Robert S. Svatek ${ }^{d}$ \\ Pierre I. Karakiewicz ${ }^{\text {e }}$ Eila Skinner ${ }^{f}$ Yves Fradet ${ }^{g}$ Jonathan I. Izawa ${ }^{\text {h }}$ Wassim Kassouf ${ }^{i}$ \\ Francesco Montorsi ${ }^{j}$ Stefan C. Müller ${ }^{b}$ Hans-Martin Fritsche ${ }^{k}$ Guru Sonpavdel Derya Tilki ${ }^{a}$ \\ Hendrik Isbarn $^{d} \quad$ Vincenzo Ficarra $^{j}$ Colin P. Dinney $^{d}$ Shahrokh F. Shariat ${ }^{\mathrm{m}}$ \\ ${ }^{a}$ Ludwig-Maximilians-Universität München, Munich, ${ }^{b}$ Universität Bonn, Bonn, Germany; ${ }^{c}$ University of Padua, \\ Padua, Italy; ${ }^{d}$ University of Texas MD Anderson Cancer Center, Houston, Tex., USA; e University of Montréal, \\ Montréal, Qué., Canada; ' University of Southern California, Los Angeles, Calif., USA; 9 Laval University, \\ Québec City, Qué., h University of Western Ontario, London, Ont., 'McGill University Health Center, Montréal, Qué., \\ Canada; Vita Salute, Milano, Italy; ${ }^{k}$ Caritas St. Josef Medical Center, Universität Regensburg, Regensburg, Germany; \\ 'Baylor College of Medicine, Houston, Tex., 'm Weill Cornell Medical College, New York, N.Y., USA
}

\section{Key Words}

Carcinoma in situ $\cdot$ Radical cystectomy, prognosis •

Bladder cancer · Urothelial carcinoma, survival

\begin{abstract}
Objective: The aim of this study was to externally validate the prognostic value of concomitant urothelial carcinoma in situ (CIS) in radical cystectomy (RC) specimens using a large international cohort of bladder cancer patients. Methods: The records of 3,973 patients treated with $\mathrm{RC}$ and bilateral lymphadenectomy for urothelial carcinoma of the bladder (UCB) at nine centers worldwide were reviewed. Surgical specimens were evaluated by a genitourinary pathologist at each center. Uni- and multivariable Cox regression models addressed time to recurrence and cancer-specific mortality after RC. Results: 1,741 (43.8\%) patients had concomitant CIS in their RC specimens. Concomitant CIS was more common in organ-confined UCB and was associated with lymphovascular invasion $(p<0.001)$. Concomitant CIS was not associ-
\end{abstract}

ated with either disease recurrence or cancer-specific death regardless of pathologic stage. The presence of concomitant CIS did not improve the predictive accuracy of standard predictors for either disease recurrence or cancer-specific death in any of the subgroups. Conclusions: We could not confirm the prognostic value of concomitant CIS in RC specimens. This, together with the discrepancy between pathologists in determining the presence of concomitant CIS at the morphologic level, limits the clinical utility of concomitant CIS in $\mathrm{RC}$ specimens for clinical decision-making.

Copyright $\odot 2011$ S. Karger AG, Basel

\section{Introduction}

Bladder cancer is the second most common malignancy of the genitourinary system [1]. Carcinoma in situ (CIS) is a potentially aggressive cancer diathesis of the bladder with varied clinical behavior. Historically, up to $83 \%$ of patients diagnosed with CIS in the absence of

\section{KARGER}

Fax +41613061234 E-Mail karger@karger.ch www.karger.com
(C) 2011 S. Karger AG, Basel

0042-1138/11/0871-0042\$38.00/0

Accessible online at:

www.karger.com/uin
Shahrokh F. Shariat, MD, PhD

Department of Urology and Medical Oncology

Brady Urologic Health Center, Weill Cornell Medical College

525 East 68th Street, Starr 900, New York, NY 10065 (USA)

Tel. +1 212746 5562, E-Mail sfshariat@gmail.com 
muscle-invasive urothelial carcinoma of the bladder (UCB) went on to develop invasive carcinoma if left untreated, and up to $39 \%$ died of their disease $[2,3]$. UCB CIS spreads along the surface of the bladder focally or in a diffuse pattern and can be difficult to diagnose. Patients presenting with isolated or concomitant CIS lesion are at high risk of experiencing disease progression to muscleinvasive UCB [4-7]. Moreover, presence of CIS is a strong predictor of UCB spread to the upper urinary tract [8-10] and the prostatic urethra [11].

We have previously shown that concomitant CIS in radical cystectomy (RC) specimens is relatively common, and patients with organ-confined UCB and concomitant CIS are at increased risk of disease recurrence and eventual death [5]. Before use in daily clinical decision-making, the prognostic value of concomitant CIS needs to be externally validated in large, multicenter datasets. Moreover, the question of whether concomitant CIS can improve the ability of established predictors of cancer outcome requires more than the conventional uni- and multivariable analyses with associated hazard rates and $\mathrm{p}$ values. In order for concomitant CIS to be clinically useful, it must add unique predictive information, thus improving the performance of a predictive model constructed without concomitant CIS by a significant margin $[12,13]$. Therefore, we sought to externally validate our previous findings in a large external, independent international cohort of patients treated with RC for UCB. We tested the hypothesis that concomitant CIS in the RC specimens could improve the accuracy of predictive models that include standard histopathologic features for prediction of stage-specific disease recurrence and survival in patients treated with $\mathrm{RC}$ for UCB.

\section{Patients and Methods}

\section{Patient Selection and Data Collection}

This was an institutional review board approved study with all participating sites providing the necessary institutional data sharing agreements prior to initiation of the study. A total of nine centers worldwide provided data. This study comprised 3,973 patients who underwent RC with bilateral lymphadenectomy between 1979 and 2008. Patients who received preoperative radiotherapy or chemotherapy were not excluded from the study. No patient had distant metastatic disease at the time of RC. None of the patients received adjuvant radiotherapy. Overall, 974 patients (24.5\%) received adjuvant chemotherapy at the investigator's discretion based on patient tumor stage and overall medical status.

A computerized databank was generated for data transfer. After combining the datasets, reports were generated for each vari- able to identify data inconsistencies and other data integrity problems. Through regular communication with all sites, resolution of all identified anomalies was achieved before analysis. Prior to final analysis, the database was frozen and the final dataset was produced for the current analysis.

\section{Pathologic Evaluation}

All surgical specimens were processed according to standard pathologic procedures, and all slides were evaluated by genitourinary pathologists according 1993 WHO grading and 2002 AJCC TNM staging. The presence of concomitant CIS was defined as the presence of CIS in conjunction with another pathologic T stage other than CIS alone. Pelvic lymph node dissections were examined grossly and all lymphoid tissue was submitted for histological examination. Lymphovascular invasion (LVI) was defined as the unequivocal presence of tumor cells within an endothelium-lined space without underlying muscular walls.

\section{Follow-Up}

Follow-up was performed according to institutional protocols. Patients were generally seen postoperatively at least every 3-4 months for the first year, semi-annually for the second year, and annually thereafter. Follow-up visits consisted of a physical examination and serum chemistry evaluation. Diagnostic imaging of the upper tracts (e.g., ultrasonography and/or intravenous pyelography, CT abdomen/pelvis with IV contrast) and chest radiography were performed at least annually or when clinically indicated. Additional radiographic evaluation, such as bone scan and/ or computerized tomography, was performed at the discretion of the treating physician. Detection of cancer in the ureter and/or urethra was coded as a second (metachronous) primary and not as local or distant recurrence. When patients died, the cause of death was determined by the treating physicians, by chart review corroborated by death certificates, or by death certificates alone. Patients who were identified as having died of bladder cancer had progressive, widely disseminated, and often highly symptomatic metastases at the time of death. Perioperative mortality (death within 30 days of surgery) was censored at the time of death for bladder cancer-specific survival analyses.

\section{Statistical Analysis}

Fisher's exact test and the $\chi^{2}$ test were used to evaluate the association between categorical variables. Differences in variables with a continuous distribution across dichotomous categories were assessed using the Mann-Whitney U test. The Kaplan-Meier method was used to calculate survival functions, and differences were assessed with the log-rank statistic. Uni- and multivariable Cox regression models addressed time to recurrence and cancerspecific mortality after RC. In all models, proportional hazards assumptions were systematically verified using the GrambschTherneau residual-based test. Since a proportion of patients treated with RC for invasive UCB die of other causes than UCB, competing risk regression was used to test the significance of variables after accounting for other-cause mortality [14]. The change in predictive accuracy (PA) was quantified with Harrell's concordance index $[15,16]$. 200-bootstrap resampling was used to adjust for overestimation $[15,16]$. All reported $p$ values are two-sided, and statistical significance was set at $\mathrm{p}<0.05$. All statistical tests were performed with S-Plus Professional (MathSoft Inc., Seattle, Wash. USA). 
Table 1. Association of concomitant CIS with clinical and pathologic characteristics in 3,973 patients treated with RC and bilateral lymphadenectomy

\begin{tabular}{|c|c|c|c|c|}
\hline & & \multicolumn{2}{|l|}{ Concomitant CIS } & \multirow[t]{2}{*}{$\mathrm{p}$} \\
\hline & & $\begin{array}{l}\text { negative } \\
(\mathrm{n}=2,232,56.2 \%)\end{array}$ & $\begin{array}{l}\text { positive } \\
(\mathrm{n}=1,741,43.8 \%)\end{array}$ & \\
\hline Age, years (median, range) & $67.0(23.3-94.2)$ & $67.0(29.7-93.9)$ & $67.1(23.4-94.2)$ & 0.230 \\
\hline $\begin{array}{l}\text { Number of lymph nodes removed } \\
\text { (median, range) }\end{array}$ & $18(0-214)$ & $16(0-122)$ & $20(0-14)$ & $<0.001$ \\
\hline \multicolumn{5}{|l|}{ Gender ${ }^{\mathrm{a}}$} \\
\hline Female & $802(20.2)$ & $469(58.5)$ & $333(41.5)$ & \\
\hline Male & $3,154(79.3)$ & $1,745(55.4)$ & $1,407(44.6)$ & 0.120 \\
\hline \multicolumn{5}{|l|}{ Pathologic stage ${ }^{a}$} \\
\hline pT0 (no tumor) & $228(5.7)$ & $228(100.0)$ & $0(0.0)$ & \\
\hline $\mathrm{pTa}$ & $129(3.2)$ & $95(73.6)$ & $34(26.4)$ & \\
\hline pT1 & $597(15.0)$ & $211(35.3)$ & $386(64.7)$ & \\
\hline pT2 & $1,063(26.8)$ & $564(53.1)$ & $499(46.9)$ & \\
\hline pT3 & $1,384(34.8)$ & $822(59.4)$ & $562(40.6)$ & \\
\hline pT4 & $572(14.4)$ & $312(54.5)$ & $260(45.5)$ & $<0.001$ \\
\hline \multicolumn{5}{|l|}{ Pathologic grade ${ }^{a}$} \\
\hline 0 (no tumor) & $228(5.7)$ & $228(100.0)$ & $0(0.0)$ & \\
\hline Low & $78(2.0)$ & $53(67.9)$ & $25(32.1)$ & \\
\hline High & $3,667(92.3)$ & $1,951(53.2)$ & $1,716(46.8)$ & $<0.001$ \\
\hline \multicolumn{5}{|l|}{ Lymphovascular invasion $^{\text {a }}$} \\
\hline Absent & $2,400(60.4)$ & $1,426(59.4)$ & $974(40.6)$ & \\
\hline Present & $1,403(35.3)$ & $732(52.2)$ & $671(47.8)$ & $<0.001$ \\
\hline \multicolumn{5}{|l|}{ Soft tissue surgical margin status ${ }^{a}$} \\
\hline Absent & $3,678(92.6)$ & $2,079(56.5)$ & $1,599(43.5)$ & \\
\hline Present & $276(6.9)$ & $139(50.4)$ & $137(49.6)$ & 0.051 \\
\hline \multicolumn{5}{|l|}{ Adjuvant chemotherapy ${ }^{\mathrm{a}}$} \\
\hline Absent & 2,997 (75.4) & $1,710(57.1)$ & $1,287(42.9)$ & \\
\hline Present & $974(24.5)$ & $521(53.5)$ & $453(46.5)$ & 0.053 \\
\hline \multicolumn{5}{|l|}{ Metastasis to lymph nodes ${ }^{a}$} \\
\hline Absent & $2,814(70.8)$ & $1,595(56.7)$ & $1,219(43.3)$ & \\
\hline Present & $1,105(27.8)$ & $592(53.6)$ & $513(46.4)$ & 0.080 \\
\hline
\end{tabular}

${ }^{a}$ Figures are number of patients with percentage in parentheses.

Gender was missing in 19 patients (0.5\%). Soft tissue surgical margin status was missing in 19 patients (0.5\%). Lymphovascular invasion was missing in 170 patients (4.3\%). Metastasis to lymph nodes was missing in 54 patients (1.4\%). Adjuvant chemotherapy was missing in 2 patients $(0.1 \%)$

\section{Results}

\section{Association of Concomitant CIS with \\ Clinicopathologic Characteristics}

The association of concomitant CIS with clinicopathologic characteristics is shown in table 1 . Of the 3,973 patients, 1,741 (43.8\%) had concomitant CIS in the RC specimens. Within pathologic stages, the proportion of concomitant CIS decreased in order from patients with pT1 $\mathrm{UCB}$, to pT2 UCB, to pT4 UCB, to pT3 UCB, and finally to pTa UCB. Patients with pathologic grades II and III were more likely to have concomitant CIS than patients with pathologic grade I $(\mathrm{p}<0.001)$. There was no difference in the rate of concomitant CIS between patients with pathologically non-muscle-invasive versus muscle-invasive UCB $(\mathrm{p}=1.000)$ and between those with pathologically non-organ-confined versus organ-confined UCB $(p=0.062)$. Patients with LVI were more likely to exhibit concomitant CIS than those without LVI $(p<0.001)$.

Association of Concomitant CIS in RC Specimens with Clinical Outcomes

Disease recurrence occurred in 1,421 of 3,973 patients (35.8\%); 1,928 patients (48.5\%) were deceased at the time 
Table 2. Pathologic stage-specific recurrence-free and cancer-specific survival estimates according to concomitant CIS in 3,973 patients treated with RC and bilateral lymphadenectomy

\begin{tabular}{|c|c|c|c|c|c|c|c|c|}
\hline \multirow{2}{*}{$\begin{array}{l}\text { Presence of } \\
\text { concomitant CIS }\end{array}$} & \multicolumn{4}{|c|}{ Probability of disease recurrence, $\%$} & \multicolumn{4}{|c|}{ Probability of cancer-specific survival, \% } \\
\hline & 3 -year $\pm \mathrm{SE}$ & 5 -year $\pm \mathrm{SE}$ & 10 -year $\pm \mathrm{SE}$ & $\mathrm{p}$ & 3 -year $\pm \mathrm{SE}$ & 5 -year $\pm \mathrm{SE}$ & 10 -year $\pm \mathrm{SE}$ & $\mathrm{p}$ \\
\hline \multicolumn{9}{|l|}{ pT1 } \\
\hline Negative & $78 \pm 3$ & $78 \pm 3$ & $75 \pm 4$ & & $83 \pm 3$ & $80 \pm 4$ & $80 \pm 4$ & \\
\hline Positive & $82 \pm 2$ & $77 \pm 3$ & $73 \pm 3$ & 0.704 & $88 \pm 2$ & $84 \pm 3$ & $78 \pm 3$ & 0.989 \\
\hline \multicolumn{9}{|l|}{$\mathrm{pT} 2$} \\
\hline Negative & $72 \pm 2$ & $68 \pm 3$ & $64 \pm 3$ & & $78 \pm 2$ & $72 \pm 3$ & $68 \pm 3$ & \\
\hline Positive & $70 \pm 3$ & $66 \pm 3$ & $66 \pm 3$ & 0.918 & $77 \pm 3$ & $72 \pm 3$ & $70 \pm 3$ & 0.845 \\
\hline \multicolumn{9}{|l|}{ pT3 } \\
\hline Negative & $51 \pm 2$ & $48 \pm 2$ & $47 \pm 2$ & & $58 \pm 2$ & $53 \pm 2$ & $50 \pm 3$ & \\
\hline Positive & $42 \pm 3$ & $38 \pm 3$ & $38 \pm 3$ & 0.018 & $48 \pm 3$ & $42 \pm 3$ & $38 \pm 3$ & 0.003 \\
\hline \multicolumn{9}{|l|}{ pT4 } \\
\hline Negative & $32 \pm 3$ & $29 \pm 3$ & $27 \pm 4$ & & $41 \pm 4$ & $34 \pm 4$ & $31 \pm 4$ & \\
\hline Positive & $35 \pm 4$ & $33 \pm 4$ & $33 \pm 4$ & 0.390 & $42 \pm 4$ & $38 \pm 4$ & $37 \pm 4$ & 0.973 \\
\hline \multicolumn{9}{|l|}{ All patients } \\
\hline Negative & $61 \pm 1$ & $59 \pm 1$ & $56 \pm 1$ & & $68 \pm 1$ & $64 \pm 1$ & $60 \pm 1$ & \\
\hline Positive & $58 \pm 1$ & $54 \pm 1$ & $52 \pm 1$ & 0.094 & $65 \pm 1$ & $60 \pm 1$ & $56 \pm 2$ & 0.058 \\
\hline \multicolumn{9}{|c|}{ Pathologic non-muscle-invasive stage $(<\mathrm{pT} 2)$} \\
\hline Negative & $87 \pm 2$ & $84 \pm 2$ & $81 \pm 3$ & & $91 \pm 2$ & $88 \pm 2$ & $87 \pm 2$ & \\
\hline Positive & $86 \pm 2$ & $82 \pm 2$ & $77 \pm 2$ & 0.614 & $92 \pm 1$ & $87 \pm 2$ & $83 \pm 2$ & 0.425 \\
\hline \multicolumn{9}{|c|}{ Pathologic muscle-invasive stage ( $\geq$ pT2) } \\
\hline Negative & $54 \pm 2$ & $51 \pm 2$ & $48 \pm 2$ & & $61 \pm 2$ & $56 \pm 2$ & $52 \pm 2$ & \\
\hline Positive & $51 \pm 2$ & $48 \pm 2$ & $47 \pm 2$ & 0.452 & $57 \pm 2$ & $52 \pm 2$ & $50 \pm 2$ & 0.155 \\
\hline \multicolumn{9}{|c|}{ Pathologic organ-confined stage $(<\mathrm{pT} 3)$} \\
\hline Negative & $81 \pm 2$ & $78 \pm 2$ & $75 \pm 2$ & & $87 \pm 1$ & $82 \pm 2$ & $79 \pm 2$ & \\
\hline Positive & $82 \pm 1$ & $79 \pm 2$ & $76 \pm 2$ & 0.571 & $88 \pm 1$ & $84 \pm 1$ & $80 \pm 2$ & 0.683 \\
\hline \multicolumn{9}{|c|}{ Pathologic non-organ-confined stage $(\geq \mathrm{pT} 3)$} \\
\hline Negative & $46 \pm 2$ & $43 \pm 2$ & $42 \pm 2$ & & $54 \pm 2$ & $49 \pm 2$ & $45 \pm 2$ & \\
\hline Positive & $41 \pm 2$ & $37 \pm 2$ & $37 \pm 2$ & 0.135 & $48 \pm 2$ & $42 \pm 2$ & $39 \pm 2$ & 0.052 \\
\hline
\end{tabular}

of analysis; 1,180 patients $(29.7 \%)$ died of bladder cancer. The median follow-up was 45 months for patients alive at last follow-up (mean \pm SD: $66.1 \pm 63.6$, interquartile range: 67). Actuarial recurrence-free survival estimates were $60 \%$ (standard error (SE): 1 ) at 3 years, 57\% (SE: 1) at 5 years, and $54 \%$ (SE: 1 ) at 10 years after RC. Actuarial cancer-specific survival estimates were $67 \%$ (SE: 1) at 3 years, $62 \%$ (SE: 1 ) at 5 years, and $58 \%$ (SE: 1 ) at 10 years after RC.

In univariable analyses, there was no difference in disease recurrence or cancer-specific survival between patients with or without concomitant CIS ( $\mathrm{p}=0.094$ and $\mathrm{p}=0.058$, respectively). We evaluated the association of concomitant CIS with bladder cancer recurrence and survival within each pathologic stage (table 2). There was no difference in disease recurrence or cancer-specific survival between patients with or without concomitant CIS in patients with pT1, pT2, pT4, organ-confined dis- ease, non-organ-confined disease, non-muscle-invasive or muscle-invasive UCB (all p values $>0.05$ ).

In patients with $\mathrm{pT} 3$ disease, patients with concomitant CIS were at increased risk of disease recurrence and cancer-specific death compared to those without concomitant CIS ( $p=0.018$ and $p=0.003$, respectively). Concomitant CIS did not retain its statistical significance when adjusted for the effects of pathologic grade, surgical margin status, LVI, and lymph node metastasis $(\mathrm{p}=0.157$ for disease recurrence and $\mathrm{p}=0.089$ for cancer-specific death).

In univariable analyses, concomitant CIS was not significantly associated with disease recurrence (HR 1.1; $\mathrm{p}=$ 0.094 ) and death (HR 1.1; $\mathrm{p}=0.058$ ). The PA of concomitant CIS for disease recurrence was 51.4\% in both all patients and organ-confined patients. The PA of concomitant CIS for cancer-specific death was $51 \%$ in all patients and $50.2 \%$ in organ-confined patients. In multivariable 
Table 3. Multivariable Cox regression analyses of concomitant CIS for prediction of recurrence and death of disease in 3,973 patients treated with RC for UBC (1,421 recurrences and 1,180 cancer-specific deaths)

\begin{tabular}{|c|c|c|c|c|c|c|}
\hline \multirow[t]{2}{*}{ Predictors } & \multicolumn{3}{|c|}{ Multivariable analysis for disease recurrence } & \multicolumn{3}{|c|}{ Multivariable analysis for cancer-related death } \\
\hline & HR & $95 \% \mathrm{CI}$ & $\mathrm{p}$ value & HR & $95 \% \mathrm{CI}$ & $\mathrm{p}$ value \\
\hline Age, continuous & 1.005 & $1.000-1.011$ & 0.06 & 1.015 & $1.008-1.021$ & $<0.001$ \\
\hline Year of surgery & & & 0.192 & & & 0.345 \\
\hline $1990-1999$ vs. $1979-1989$ & 0.924 & $0.778-1.098$ & 0.368 & 0.917 & $0.764-1.100$ & 0.350 \\
\hline 2000-2008 vs. $1979-1989$ & 1.031 & $0.862-1.233$ & 0.737 & 1.006 & $0.828-1.223$ & 0.953 \\
\hline Pathologic T stage & & & $<0.001$ & & & $<0.001$ \\
\hline pTa vs. pT0 & 1.486 & $0.742-2.975$ & 0.263 & 0.964 & $0.364-2.552$ & 0.941 \\
\hline pT1 vs. pT0 & 1.855 & $1.165-2.954$ & 0.009 & 1.067 & $0.412-2.765$ & 0.894 \\
\hline pT2 vs. pT0 & 2.587 & $1.672-4.003$ & $<0.001$ & 1.514 & $0.590-3.890$ & 0.389 \\
\hline pT3 vs. pT0 & 4.002 & $2.599-6.163$ & $<0.001$ & 2.526 & $0.986-6.470$ & 0.054 \\
\hline pT4 vs. pT0 & 4.973 & $3.188-7.756$ & $<0.001$ & 3.003 & $1.169-7.715$ & 0.022 \\
\hline Lymphovascular invasion & 1.442 & $1.273-1.633$ & $<0.001$ & 1.446 & $1.262-1.656$ & $<0.001$ \\
\hline Concomitant CIS & 0.997 & $0.889-1.118$ & 0.960 & 0.986 & $0.869-1.120$ & 0.833 \\
\hline Soft tissue surgical margin & 1.524 & $1.267-1.834$ & $<0.001$ & 1.746 & $1.439-2.118$ & $<0.001$ \\
\hline Pathological grade & & & 0.226 & & & 0.271 \\
\hline Grade 2 vs. grade 1 & 1.611 & $0.834-3.112$ & 0.116 & 1.867 & $0.850-4.104$ & 0.120 \\
\hline Grade 3 vs. grade 1 & 1.703 & $0.878-3.303$ & 0.368 & 1.803 & $0.816-3.983$ & 0.145 \\
\hline Lymph node metastasis & 1.783 & $1.563-2.035$ & $<0.001$ & 2.009 & $1.739-2.321$ & $<0.001$ \\
\hline Number of removed lymph nodes & 0.999 & $0.996-1.002$ & 0.557 & 1 & $0.996-1.004$ & 0.989 \\
\hline Number of positive lymph nodes & 1.021 & $1.012-1.030$ & $<0.001$ & 1.024 & $1.014-1.034$ & $<0.001$ \\
\hline
\end{tabular}

analyses, concomitant CIS was not associated with disease recurrence (HR 0.99; $\mathrm{p}=0.960$ ) or death (HR 0.99; $\mathrm{p}=0.833$ ) when adjusted for the effects of standard pathologic features (table 3). Addition of concomitant CIS to multivariable models that included standard pathologic features improved its PA by $0.1 \%$ for disease recurrence and $0 \%$ for cancer-specific death. In patients with organconfined disease, addition of concomitant CIS to multivariable models that included standard pathologic features (pathologic stage, grade, and LVI) improved its PA by $0.1 \%$ for disease recurrence and $0.2 \%$ for cancer-specific death.

\section{Discussion}

Urothelial CIS is likely the most common precursor of invasive UCB [17-19]. Several cytogenetic [17, 20] molecular genetic [21] and immunohistologic [22-25] studies have shown similar molecular alterations for CIS and muscle-invasive UCB. Several small studies have shown that the presence of concomitant CIS in patients with papillary, non-muscle-invasive UCB is associated with significantly worse clinical outcome after RC [26-28]. In patients with clinical stage T1 grade 3 UCB treated with
RC, concomitant CIS was the only pre-cystectomy factor associated with disease recurrence and mortality in 171 patients treated with RC [7]. The risk of disease recurrence increased 2.5-fold and the risk of bladder cancerspecific death increased 3-fold when concomitant CIS was identified preoperatively. Similarly, Masood et al. [26] reported that T1 grade 3 UCB patients with concomitant CIS have a higher probability of disease progression than those without concomitant CIS (55 vs. $6 \%$ ). Moreover, two studies have also shown that CIS is an independent predictor of disease progression to muscle-invasive UCB [29, 30]. Sylvester et al. [30] studied 2,596 patients with Ta and T1 bladder cancer enrolled in EORTC clinical trials and found a 3.4-fold risk of progression to muscle-invasive disease among patients with CIS. Thus, patients with non-muscle-invasive disease with CIS are at a high risk of progression to muscle invasion and also have a worse prognosis after RC. This has led many clinicians to follow CIS-only patients closely and consider them for early RC.

The prognostic value of concomitant CIS in patients with papillary tumors remains unclear to date. Recently, in a tri-institutional study of 713 patients treated with RC, we found that concomitant CIS is more common in lower-stage and higher-grade disease, and is significantly as- 
sociated with UCB involvement of the urethra at RC. More importantly, the presence of concomitant CIS in patients with pathologic non-muscle-invasive UCB treated with RC was associated with a significantly worse outcome [5]. However, before inclusion into daily clinical decision-making, such findings need to be confirmed in a large, multi-institutional, independent dataset. Therefore, to validate the clinical utility of concomitant CIS in $\mathrm{RC}$ specimens for indentifying patients at high risk for disease recurrence who could benefit from close surveillance or inclusion into a predictive tool that would help select patients for adjuvant therapy, we attempted to validate our previous findings in an independent, international cohort of almost 4,000 RC patients.

We confirmed that many patients harbor concomitant CIS (43.8\%) in addition to their papillary UCB in the RC specimens. Moreover, concomitant CIS was significantly more common in low-stage and high-grade UCB and was associated with LVI. While association with features of aggressive disease is important, prediction of outcomes after RC is more important for the management of UCB patients. In this context, differences in clinical outcome between patients with and without concomitant CIS could not only be related to tumor progression and development of metastases due to more aggressive tumor biology, but could also be caused by a result of intraluminal recurrence on the basis of multifocal disease. We found no association between concomitant CIS and clinical outcomes after RC in the entire population or in stage subgroups. Although the presence of concomitant CIS was associated with increased risk of disease recurrence and cancer-specific death for patients with pT3 disease, this phenomenon did not retain its statistical significance when adjusted for the effects of pathologic grade, surgical margin status, LVI and lymph node metastasis. Concomitant CIS did not add any information beyond standard pathologic features for prediction of oncologic outcomes after RC. This remained true in single-center analyses (no change in PA; data not shown). This lack of prognostic value was seen in standard multivariable and PA analyses. Concomitant CIS, thus, does not add any additional information for management of UCB patients and should not be used for clinical decision-making.

This study suffers from several limitations. RC was performed by various surgeons and specimens were reviewed by multiple pathologists. The difficulty and dedication necessary in determining its presence of concomitant CIS at the morphologic level may have resulted in differences between pathologists specimens (interobserver variability). While this variability may be con- strued as limitations, the role of an external validation is to see whether a factor retains its value in different cohorts of patients representing a real-world scenario. While these limitations can be construed as significant, the purpose of this study was to reflect a real-world practice in which multiple pathologists review tissue specimens and their interpretation is used in clinical decisionmaking with the patient. Indeed, the large number of patients and the diversity of centers in expertise, volume, and geographical location increase the generalizability of the findings.

In this multicenter study, more than half of all patients undergoing RC for UCB had concomitant CIS on final pathology. Concomitant CIS was more common in organ-confined UCB and high-grade UCB, and concomitant CIS was associated with LVI. However, we could not confirm the prognostic value of concomitant CIS in the $\mathrm{RC}$ specimens for prediction of clinical outcomes. Concomitant CIS had no impact on disease recurrence or cancer-specific survival in standard Cox regression and PA analyses. These data support that CIS concomitant with papillary disease in the RC specimens does not add any information beyond standard histopathologic features and has no clinical utility in the management of UCB patients.

\section{Disclosure Statement}

The authors have no conflicts of interest to declare.

References

1 Jemal A, Siegel R, Ward E, Hao Y, Xu J, Thun MJ: Cancer statistics, 2009. CA Cancer J Clin 2009;59:225-249.

2 Skinner DG, Richie JP, Cooper PH, Waisman J, Kaufman JJ: The clinical significance of carcinoma in situ of the bladder and its association with overt carcinoma. J Urol 1974; 112:68-71.

-3 Utz DC, Hanash KA, Farrow GM: The plight of the patient with carcinoma in situ of the bladder. J Urol 1970;103:160-164.

4 Lamm DL: Carcinoma in situ. Urol Clin North Am 1992;19:499-508.

5 Shariat SF, Palapattu GS, Karakiewicz PI, Rogers CG, Vazina A, Bastian PJ, et al: Concomitant carcinoma in situ is a feature of aggressive disease in patients with organ-confined TCC at radical cystectomy. Eur Urol 2007;51:152-160. 
6 Shariat SF, Palapattu GS, Amiel GE, Karakiewicz PI, Rogers CG, Vazina A, et al: Characteristics and outcomes of patients with carcinoma in situ only at radical cystectomy. Urology 2006;68:538-542.

7 Gupta A, Lotan Y, Bastian PJ, Palapattu GS, Karakiewicz PI, Raj GV, et al: Outcomes of patients with clinical T1 grade 3 urothelial cell bladder carcinoma treated with radical cystectomy. Urology 2008;71:302-307.

$>8$ Zincke H, Garbeff PJ, Beahrs JR: Upper urinary tract transitional cell cancer after radical cystectomy for bladder cancer. J Urol 1984;131:50-52.

>9 Solsona E, Iborra I, Ricos JV, Dumont R, Casanova JL, Calabuig C: Upper urinary tract involvement in patients with bladder carcinoma in situ (Tis): its impact on management. Urology 1997;49:347-352.

-10 Millan-Rodriguez F, Chechile-Toniolo G, Salvador-Bayarri J, Huguet-Perez J, VicenteRodriguez J: Upper urinary tract tumors after primary superficial bladder tumors: prognostic factors and risk groups. J Urol 2000;164:1183-1187.

-11 Nixon RG, Chang SS, Lafleur BJ, Smith JJ, Cookson MS: Carcinoma in situ and tumor multifocality predict the risk of prostatic urethral involvement at radical cystectomy in men with transitional cell carcinoma of the bladder. J Urol 2002;167:502-505.

12 Kattan MW: Judging new markers by their ability to improve predictive accuracy. J Natl Cancer Inst 2003;95:634-635.

-13 Shariat SF, Karakiewicz PI, Ashfaq R, Lerner SP, Palapattu GS, Cote RJ, et al: Multiple biomarkers improve prediction of bladder cancer recurrence and mortality in patients undergoing cystectomy. Cancer 2008;112:315325.
14 Fine JP: Regression modeling of competing crude failure probabilities. Biostatistics 2001;2:85-97.

15 Harrell FE Jr, Califf RM, Pryor DB, Lee KL, Rosati RA: Evaluating the yield of medical tests. JAMA 1982;247:2543-2546.

16 Harrell FE Jr, Lee KL, Mark DB: Multivariable prognostic models: issues in developing models, evaluating assumptions and adequacy, and measuring and reducing errors. Stat Med 1996;15:361-387.

17 Habuchi T, Ogawa O, Kakehi Y, Ogura K, Koshiba M, Sugiyama T, et al: Allelic loss of chromosome 17p in urothelial cancer: strong association with invasive phenotype. J Urol 1992;148:1595-1599.

18 Spruck CH 3rd, Ohneseit PF, Gonzalez-Zulueta M, Esrig D, Miyao N, Tsai YC, et al: Two molecular pathways to transitional cell carcinoma of the bladder. Cancer Res 1994;54: 784-788.

19 Rosin MP, Cairns P, Epstein JI, Schoenberg MP, Sidransky D: Partial allelotype of carcinoma in situ of the human bladder. Cancer Res 1995;55:5213-5216.

20 Knowles MA, Elder PA, Williamson M, Cairns JP, Shaw ME, Law MG: Allelotype of human bladder cancer. Cancer Res 1994;54: 531-538.

21 Dyrskjot L, Kruhoffer M, Thykjaer T, Marcussen N, Jensen JL, Moller K, et al: Gene expression in the urinary bladder: a common carcinoma in situ gene expression signature exists disregarding histopathological classification. Cancer Res 2004;64:4040-4048.

22 Sarkis AS, Dalbagni G, Cordon-Cardo C, Melamed J, Zhang ZF, Sheinfeld J, et al: Association of p53 nuclear overexpression and tumor progression in carcinoma in situ of the bladder. J Urol 1994;152:388-392.

-23 Shariat SF, Pahlavan S, Baseman AG, Brown RM, Green AE, Wheeler TM, et al: E-cadherin expression predicts clinical outcome in carcinoma in situ of the urinary bladder Urology 2001;57:60-65.
24 Shariat SF, Kim JH, Ayala GE, Kho K, Wheeler TM, Lerner SP: Cyclooxygenase-2 is highly expressed in carcinoma in situ and T1 transitional cell carcinoma of the bladder. J Urol 2003;169:938-942.

-25 Shariat SF, Kim J, Raptidis G, Ayala GE, Lerner SP: Association of p53 and p21 expression with clinical outcome in patients with carcinoma in situ of the urinary bladder. Urology 2003;61:1140-1155.

-26 Masood S, Sriprasad S, Palmer JH, Mufti GR: T1G3 bladder cancer - indications for early cystectomy. Int Urol Nephrol 2004;36:4144.

27 Vicente J, Laguna MP, Duarte D, Algaba F, Chechile G: Carcinoma in situ as a prognostic factor for G3pT1 bladder tumours. Br J Urol 1991;68:380-382.

28 Smits G, Schaafsma E, Kiemeney L, Caris C, Debruyne F, Witjes JA: Microstaging of pT1 transitional cell carcinoma of the bladder: identification of subgroups with distinct risks of progression. Urology 1998;52:10091013.

-29 Orsola A, Trias I, Raventos CX, Espanol I, Cecchini L, Bucar S, et al: Initial high-grade T1 urothelial cell carcinoma: feasibility and prognostic significance of lamina propria invasion microstaging $(\mathrm{T} 1 \mathrm{a} / \mathrm{b} / \mathrm{c})$ in BCG-treated and BCG-non-treated patients. Eur Urol 2005;48:231-238.

30 Sylvester RJ, van der Meijden AP, Oosterlinck W, Witjes JA, Bouffioux C, Denis L, et al: Predicting recurrence and progression in individual patients with stage Ta T1 bladder cancer using EORTC risk tables: a combined analysis of 2,596 patients from seven EORTC trials. Eur Urol 2006;49:466-465. 